\title{
Canonical description of T-duality with NSNS background
}

\section{J. Klusoň}

Department of Theoretical Physics and Astrophysics, Faculty of Science, Masaryk University, Kotlárská 2, 611 3\%, Brno, Czech Republic

E-mail: klu@physics.muni.cz

ABSTRACT: We study T-duality with non-zero components of NSNS two form field along directions we dualize with the help of canonical formalism. As a result of this procedure we determine generalized Buscher's rules. We also apply the same procedure to the case of non-relativistic string.

KEYwords: Bosonic Strings, String Duality

ArXiv EPrint: 1911.13001 


\section{Contents}

1 Introduction and summary 1

2 T-duality with NSNS two form in canonical formalism 2

$\begin{array}{llr}3 & \text { T-duality of non-relativistic string } & 6\end{array}$

\section{Introduction and summary}

One of the most important properties of string theory is T-duality, for earlier review, see [1]. This is duality of string theory whose most powerful description is given in terms of Buscher's rules [2,3] of the transformations of the background fields under T-duality. More explicitly, we start with string sigma model on the background where the background metric possesses one isometry, at least. Then we gauge this isometry so that this is now local symmetry on the string world-sheet when we introduce corresponding covariant derivative and two dimensional gauge field which are non-propagating. In order to ensure that these fields are not dynamical we add to the action term that ensures that the field strength of this gauge field is zero. As the next step we fix the gauge when we take the world-sheet mode that parameterizes direction with gauged isometry, to be zero. Then we can solve the flatness of the gauge field with introducing new scalar mode that parameterizes the string propagating along dual coordinate where now the background fields are related to the original ones through Buscher's rules.

While this procedure is well established in case of the T-duality along one direction situation when we have several isometry directions with non-trivial NSNS two form is more complicated. In our previous paper [4] we encountered this problem when we analyzed Tduality of non-relativistic string in torsional Newton-Cartan background. Since such a string can be defined as T-dual of the relativistic string in the background with light-like isometry [5-8] in order to study its T-duality properties we had to analyze T-duality of relativistic string along two directions. Even if such a procedure seems to be straightforward it turned out to be rather involved and we were not able to determine transformations rules in the general case of non-zero components of NSNS two form along directions we dualize. Surprisingly we were not able to find corresponding transformations rules in the literature and the goal of this paper is to find them.

Explicitly, we would like to study general string theory action in the background with non-trivial metric and NSNS two form with isometry along several directions. In principle we could start with the Lagrangian formulation and gauge corresponding isometry directions and then proceed as in the case of single isometry direction. However now the situation is much more intricate due to the presence of non-trivial NSNS two form that now appears in corresponding equations of motions. It is important to stress that expressions with 
NSNS two form are multiplied with world-sheet antisymmetric symbol while expressions with target space metric are multiplied with world-sheet metric so that it is very difficult to solve them. However there is an alternative way how to study T-duality which is canonical approach to T-duality $[9,10]$. This procedure is based on the Hamiltonian form of string in the background that possesses an isometry. Then we perform canonical transformations of the coordinate that labels this isometric direction and we derive new Hamiltonian for dual theory. Finally we perform inverse Legendre transformations to T-dual Lagrangian and we determine dual background fields whose forms agree with Buscher's rules. We use this procedure to the case of the relativistic string in the background with non-zero NSNS two form. We determine T-dual Hamiltonian which is straightforward procedure. On the other hand in order to determine corresponding transformation rules for the background field we have to find T-dual Lagrangian. It turns out that this is non-trivial procedure that deserves careful treatment. After performing this analysis we obtain generalized Buscher's rules that also include background NSNS two form. As far as we know these transformations were not determined before in the full generality.

As the next goal of this work is to analyze T-duality of non-relativistic string in torsional Newton-Cartan background and with non-trivial NSNS two form. This section is generalization of the analysis performed recently in [4]. We show that generally under T-duality non-relativistic string maps to the relativistic one with specific form of the background fields and we also analyze conditions that determine that non-relativistic string maps to non-relativistic string again. We show that these conditions are the same as ones that were found in [4].

Let us outline our result and suggest possible extension of this work. We study Tduality along several directions with non-zero NSNS two form in the framework of canonical formalism. We obtain corresponding generalized Buscher's rules which as far as we know, were determined for the first time in this generality. Then we analyze T-duality of nonrelativistic string in torsional Newton-Cartan background with non-zero NSNS two form and we determined conditions under such a string maps to non-relativistic string. We mean that the analysis presented in this paper could be extended in several directions. In particular, it would be nice to study whether T-dual Hamiltonian found in this paper could be useful for the symmetric formulation of the string in the Double string theory. It would be also nice whether similar analysis could be performed in case of the non-relativistic string. We hope to return to these problems in future.

The structure of this paper is as follows. In the next section 2 we analyze T-duality of relativistic string with the help of canonical formalism. Then in section 3 we present the same analysis in case of the non-relativistic string in torsional Newton-Cartan background.

\section{T-duality with NSNS two form in canonical formalism}

T-duality with non-trivial NSNS two form along directions we dualize is remarkably complex if we consider general bosonic string. The reason is that the term which is proportional to the embedding of the target space metric is multiplied by word-sheet metric while term which is proportional to NSNS two form is multiplied by world-sheet antisymmetric tensor. 
Then it is very difficult to solve equations of motion for auxiliary world-sheet gauge fields. However the situation simplifies considerably when we analyze T-duality with the help of the canonical formalism $[9,10]$. To do this we have to find Hamiltonian for bosonic string with the action

$$
S=-T \int d^{2} \sigma \sqrt{-\gamma} \gamma^{\alpha \beta} G_{M N} \partial_{\alpha} x^{M} \partial_{\beta} x^{N}-\frac{T}{2} \int d^{2} \sigma \epsilon^{\alpha \beta} B_{M N} \partial_{\alpha} x^{M} \partial_{\beta} x^{m},
$$

where $T$ is string tensor, $\gamma_{\alpha \beta}$ is world-sheet metric, $x^{M}, M=0, \ldots, 25$ parameterize embedding of the string in the target space background with the metric $G_{M N}$ and NSNS two form $B_{M N}$. Further, world-sheet is parameterized with $\sigma^{\alpha}, \alpha=0,1, \sigma^{0}=\tau, \sigma^{1}=\sigma$ and $\epsilon^{\alpha \beta}=-\epsilon^{\beta \alpha}$ is antisymmetric tensor.

Since we are going to analyze T-duality with the help of the canonical formalism we have to introduce Hamiltonian for bosonic string. The result is well known and we write the result as

$$
H=\int d \sigma\left(N^{\tau} \mathcal{H}_{\tau}+N^{\sigma} \mathcal{H}_{\sigma}\right)
$$

where

$$
\begin{aligned}
& \mathcal{H}_{\tau}=\Pi_{M} G^{M N} \Pi_{N}+T^{2} G_{M N} \partial_{\sigma} x^{M} \partial_{\sigma} X^{N}, \quad \Pi_{M}=p_{M}+T B_{M N} \partial_{\sigma} x^{N}, \\
& \mathcal{H}_{\sigma}=p_{M} \partial_{\sigma} x^{N},
\end{aligned}
$$

where $N^{\tau}, N^{\sigma}$ are Lagrange multipliers corresponding to the first class constraints $\mathcal{H}_{\tau} \approx 0, \mathcal{H}_{\sigma} \approx 0$.

Now we are ready to proceed to the canonical description of T-duality when we select $2 p$ coordinates $x^{m}, m, n=25-2 p, \ldots, 25$ and the remaining ones $\mu, \nu=0,1, \ldots, 2 p-1$ and dualize along $x^{m}$ directions. According to $[9,10]$ such a duality can be considered as canonical transformation. Explicitly, we introduce T-dual variables $\tilde{x}_{m}$ and corresponding conjugate momenta $\tilde{p}^{m}$. Then the canonical transformations have the form

$$
\tilde{p}^{m}=-T \partial_{\sigma} x^{m}, \quad p_{m}=-T \partial_{\sigma} \tilde{x}_{m}
$$

and T-dual Hamiltonian arises when we replace original variables $p_{m}, x^{m}$ in the Hamiltonian constraint with T-dual ones given above. As a result we obtain following T-dual Hamiltonian constrain in the form

$$
\begin{aligned}
\mathcal{H}_{\tau}^{T}= & \left(k_{\mu}-B_{\mu m} \tilde{p}^{m}\right) G^{\mu \nu}\left(k_{\nu}-B_{\nu n} \tilde{p}^{n}\right)+2\left(k_{\mu}-B_{\mu m} \tilde{p}^{m}\right) G^{\mu n}\left(-T \partial_{\sigma} \tilde{x}_{n}-B_{n m} \tilde{p}^{m}+T B_{n \mu} \partial_{\sigma} x^{\mu}\right) \\
& +\left(-T \partial_{\sigma} \tilde{x}_{m}-B_{m k} \tilde{p}^{k}+T B_{m \mu} \partial_{\sigma} x^{\mu}\right) G^{m n}\left(-T \partial_{\sigma} \tilde{x}_{n}-B_{n l} \tilde{p}^{l}+T B_{n \nu} \partial_{\sigma} x^{\nu}\right) \\
& +T^{2} \partial_{\sigma} x^{\mu} G_{\mu \nu} \partial_{\sigma} x^{\nu}-2 T \tilde{p}^{m} G_{m \mu} \partial_{\sigma} x^{\mu}+\tilde{p}^{m} G_{m n} \tilde{p}^{n} \\
= & k_{\mu} G^{\mu \nu} k_{\nu}+2 \tilde{p}^{m} B_{m M} G^{M \mu} k_{\mu}+\tilde{p}^{m} \mathbf{H}_{m n} \tilde{p}^{n}+2 T k_{\mu} G^{\mu n} \mathbf{V}_{n} \\
& +T^{2} \mathbf{V}_{m} G^{m n} \mathbf{V}_{n}+2 T \tilde{p}^{k}\left(B_{k M} G^{M n} \mathbf{V}_{n}-G_{m \mu} \partial_{\sigma} x^{\mu}\right)+T^{2} \partial_{\sigma} x^{\mu} G_{\mu \nu} \partial_{\sigma} x^{\nu}
\end{aligned}
$$

where

$$
k_{\mu}=p_{\mu}+T B_{\mu \nu} \partial_{\sigma} x^{\nu}, \quad \mathbf{V}_{n}=-\partial_{\sigma} \tilde{x}_{n}+B_{n \mu} \partial_{\sigma} x^{\mu}
$$


and where we used the fact that under T-duality (2.4) $\Pi_{M}$ transform as

$$
\Pi_{\mu}=k_{\mu}-B_{\mu m} \tilde{p}^{m}, \quad \Pi_{m}=-T \partial_{\sigma} \tilde{x}_{m}-B_{m n} \tilde{p}^{n}+T B_{m \mu} \partial_{\sigma} x^{\mu} .
$$

As the last important step we introduced matric $\mathbf{H}_{m n}$ defined as

$$
\mathbf{H}_{m n}=G_{m n}-B_{m M} G^{M N} B_{N m} .
$$

In order to determine transformation rules for metric and NSNS two form field under these T-duality transformations we have to find Lagrangian for T-dual string. With the help of the Hamiltonian constraint (2.5) we obtain following equation of motion

$$
\begin{aligned}
\partial_{\tau} \tilde{x}_{m}=\left\{\tilde{x}_{m}, H\right\}= & 2 N^{\tau} B_{m M} G^{M \mu} k_{\mu}+2 N^{\tau} \mathbf{H}_{m n} \tilde{p}^{n} \\
& +2 T N^{\tau}\left(B_{m M} G^{M n} \mathbf{V}_{n}-G_{m \mu} \partial_{\sigma} x^{\mu}\right)+N^{\sigma} \partial_{\sigma} \tilde{x}_{m}, \\
\partial_{\tau} x^{\mu}=\left\{x^{\mu}, H\right\}= & 2 N^{\tau} G^{\mu \nu} k_{\nu}-2 N^{\tau} G^{\mu M} B_{M m} \tilde{p}^{m}+2 N^{\tau} T G^{\mu n} \mathbf{V}_{n} .
\end{aligned}
$$

From the second equation we express $k_{\mu}$ as

$$
k_{\mu}=\frac{1}{2 N^{\tau}} \hat{G}_{\mu \nu}\left(X^{\nu}+2 N^{\tau} G^{\nu M} B_{M m} \tilde{p}^{m}-2 N^{\tau} T G^{\mu n} \mathbf{V}_{n}\right)
$$

where

$$
\hat{G}_{\mu \nu}=G_{\mu \nu}-G_{\mu m} \hat{G}^{m n} G_{n \nu}, \quad \tilde{X}^{\mu}=\partial_{\tau} x^{\mu}-N^{\sigma} \partial_{\sigma} x^{\mu}, \quad \tilde{X}_{m}=\partial_{\tau} \tilde{x}_{m}-N^{\sigma} \partial_{\sigma} \tilde{x}_{m},
$$

and where we introduced matrix $\hat{G}^{m n}$ that is inverse to the matrix $G_{m n}$

$$
\hat{G}^{m n} G_{n k}=\delta_{k}^{m} \text {. }
$$

Further, matrix $\hat{G}_{\mu \nu}$ has following important properties

$$
\hat{G}_{\mu \nu} G^{\nu \rho}=\delta_{\mu}^{\rho}, \quad \hat{G}_{\mu \nu} G^{\nu m}=-G_{\mu m} \hat{G}^{m n} .
$$

Then inserting (2.10) into the first equation in (2.9) we obtain

$$
\tilde{X}_{m}=2 N^{\tau} \hat{G}_{m n} \tilde{p}^{n}+2 N^{\tau} T B_{m l} \hat{G}^{l n} \mathbf{V}_{n}+B_{m \mu} X^{\mu}-B_{m n} \hat{G}^{n k} G_{k \nu} X^{\nu}-2 T N^{\tau} G_{m \mu} \partial_{\sigma} x^{\mu},
$$

where we used following important properties

$$
\begin{aligned}
& B_{m M} G^{M \mu} \hat{G}_{\mu \nu} G^{\nu N} B_{N n}=B_{m M} G^{M N} B_{N n}-B_{m k} \hat{G}^{k l} B_{l n}, \\
& -B_{m M} G^{M \mu} \hat{G}_{\mu \nu} G^{\nu n} \mathbf{V}_{n}=B_{m l} \hat{G}^{l n} \mathbf{V}_{n}-B_{m M} G^{M n} \mathbf{V}_{n},
\end{aligned}
$$

and where we defined

$$
\hat{G}_{m n}=G_{m n}-B_{m k} \hat{G}^{k l} B_{l n} .
$$

Now it is easy to express $\tilde{p}^{m}$ from (2.14)

$$
\tilde{p}^{m}=\frac{1}{2 N^{\tau}} \tilde{G}^{m n}\left(\tilde{X}_{n}-B_{n \mu} X^{\mu}+B_{n k} \hat{G}^{k l} G_{l \nu} X^{\nu}+2 T N^{\tau} G_{m \mu} \partial_{\sigma} x^{\mu}-2 N^{\tau} T B_{m l} \hat{G}^{l n} \mathbf{V}_{n}\right),
$$


where $\tilde{G}^{m n}$ is matrix inverse to $\hat{G}_{m n}$ defined as

$$
\tilde{G}^{m n} \hat{G}_{n k}=\delta_{k}^{m} \text {. }
$$

An existence of the matric $\tilde{G}^{m n}$ is crucial consequence of the presence of non-trivial components of NSNS two form $B_{m n}$. Clearly for $B_{m n}=0, \tilde{G}^{m n}$ reduces to $\hat{G}^{m n}$. Now we are ready to find corresponding Lagrangian density

$$
\begin{aligned}
\mathcal{L}= & p_{\mu} \partial_{\tau} x^{\mu}+\tilde{p}^{m} \partial_{\tau} \tilde{x}_{m}-N^{\tau} \mathcal{H}_{\tau}^{T}-N^{\sigma} \mathcal{H}_{\sigma}^{T} \\
= & \frac{1}{4 N^{\tau}}\left[X^{\mu} G_{\mu \nu}^{\prime} X^{\nu}+\tilde{X}_{m} G^{\prime m n} \tilde{X}_{n}+X^{\mu} G_{\mu}^{\prime}{ }^{m} \tilde{X}_{m}+\tilde{X}_{n} G_{\nu}^{\prime n} X^{\nu}\right] \\
& -T^{2} N^{\tau}\left[\partial_{\sigma} \tilde{x}_{m} G^{\prime m n} \partial_{\sigma} \tilde{x}_{n}+\partial_{\sigma} x^{\mu} G_{\mu \nu}^{\prime} \partial_{\sigma} x^{\nu}+\partial_{\sigma} \tilde{x}_{m} G^{\prime m}{ }_{\nu} \partial_{\sigma} x^{\nu}+\partial_{\sigma} x^{\mu} G_{\mu}^{\prime n} \partial_{\sigma} \tilde{x}_{n}\right] \\
& -T B_{\mu \nu}^{\prime} \partial_{\tau} x^{\mu} \partial_{\sigma} x^{\nu}-T B_{\mu}^{\prime n} \partial_{\tau} x^{\mu} \partial_{\sigma} \tilde{x}_{n}-T B_{\nu}^{\prime m} \partial_{\tau} \tilde{x}_{m} \partial_{\sigma} \tilde{x}^{\nu}-T B^{\prime m n} \partial_{\tau} \tilde{x}_{m} \partial_{\sigma} \tilde{x}_{n} \\
\equiv & \frac{1}{4 N^{\tau}}\left[g_{\tau \tau}^{\prime}-2 N^{\sigma} g_{\tau \sigma}^{\prime}+\left(N^{\sigma}\right)^{2} g_{\sigma \sigma}^{\prime}\right]-T^{2} N^{\tau} g_{\sigma \sigma}^{\prime}-T b_{\tau \sigma}^{\prime},
\end{aligned}
$$

where we used an important property of the matrix $\tilde{G}^{m n}$

$$
\tilde{G}^{m n}=\hat{G}^{m n}+\hat{G}^{m k} B_{k l} \tilde{G}^{l p} B_{p r} \hat{G}^{r n}, \quad \tilde{G}^{m n} B_{n k} \hat{G}^{k l}=\hat{G}^{m n} B_{n k} \tilde{G}^{k l} .
$$

Finally the components of T-dual metric and NSNS two form have the form

$$
\begin{aligned}
G_{\mu \nu}^{\prime} & =G_{\mu \nu}-G_{\mu m} \hat{G}^{m n} G_{n \nu}-\left(B_{\mu m}-G_{\mu l} \hat{G}^{l k} B_{k m}\right) \tilde{G}^{m n}\left(B_{n \nu}-B_{n k^{\prime}} \hat{G}^{k^{\prime} l^{\prime}} G_{l^{\prime} \nu}\right), \\
G^{\prime m n} & =\tilde{G}^{m n}, \\
G^{\prime m} & =-\tilde{G}^{m n}\left(B_{n \nu}-B_{n k} \hat{G}^{k l} G_{l \nu}\right), \\
G_{\mu}^{\prime}{ }^{n} & =\left(B_{\mu n}-G_{\mu k} \hat{G}^{k l} B_{l m}\right) \tilde{G}^{m n} . \\
B^{\prime m n} & =\tilde{G}^{m k} B_{k l} \hat{G}^{l n}, \\
B_{\mu \nu}^{\prime} & =B_{\mu \nu}+G_{\mu m} \tilde{G}^{m n} B_{n \nu}-B_{\mu m} \tilde{G}^{m n} G_{n \nu}+B_{\mu m} \tilde{G}^{m n} B_{n l} \hat{G}^{l k} B_{k \nu}+G_{\mu k} \hat{G}^{k l} B_{l m} \tilde{G}^{m n} G_{n \nu}, \\
B_{\mu}^{\prime n} & =\left[G_{\mu m}-B_{\mu k} \hat{G}^{k l} B_{l m}\right] \tilde{G}^{m n}, \\
B_{\mu}^{\prime m} & =\tilde{G}^{m n}\left[G_{n \mu}-B_{n k} \hat{G}^{k l} B_{l \mu}\right] .
\end{aligned}
$$

These are most general T-duality transformation rules in case of non-zero components of NSNS two form along directions where T-duality is performed. We see presence of two inverse metrics $\hat{G}^{m n}$ and $\tilde{G}^{m n}$ where $\tilde{G}^{m n}$ reduces into $\hat{G}^{m n}$ in case of zero $B_{m n}$. In fact, in this case the transformation rules reduce to transformation rules found in the previous paper [4]. Finally $g_{\alpha \beta}^{\prime}, b_{\alpha \beta}^{\prime}$ introduced on the last line in (2.19) are pullbacks of T-dual components of metric and NSNS two form given in (2.21) to the string's world-sheet. Finally, if we integrate out $N^{\tau}, N^{\sigma}$ we obtain

$$
N^{\sigma}=\frac{g_{\tau \sigma}^{\prime}}{g_{\sigma \sigma}^{\prime}}, \quad N^{\tau}=\frac{1}{2 T} \frac{\sqrt{-\operatorname{det} g_{\alpha \beta}^{\prime}}}{g_{\sigma \sigma}^{\prime}} .
$$

Then inserting these results back to (2.19) we obtain Nambu-Goto form of the string action in T-dual background (2.21)

$$
\mathcal{L}=-T \sqrt{-\operatorname{det} g_{\alpha \beta}^{\prime}}-T b_{\tau \sigma}^{\prime} .
$$

In the next section we focus on T-duality with non-zero NSNS two form in case of nonrelativistic string. 


\section{T-duality of non-relativistic string}

In this section we will briefly discuss T-duality properties of non-relativistic string in torsional Newton-Cartan background with the presence of non-zero NSNS two form. Analysis presented in this section is generalization of our previous paper [4] so that we recommend this paper for more details. As was argued previously in [5-8] non-relativistic string in torsional Newton-Cartan background can be naturally defined with the help of T-duality along light-like direction of relativistic string. Since as we argued in [11] such a transformation is problematic it is natural to consider an extended action with two auxiliary fields $\lambda^{+}, \lambda^{-}[12]$. This extended action is chosen in such a way that when we solve equation of motion for $\lambda^{+}, \lambda^{-}$we obtain original relativistic string with light-like isometry. Explicitly, let us consider following Lagrangian density

$$
\begin{aligned}
\mathcal{L}=-\frac{T}{2} N \sqrt{\omega}[ & -\nabla_{n} x^{M} G_{M N} \nabla_{n} x^{N}+\frac{1}{\omega} \partial_{\sigma} x^{M} \partial_{\sigma} x^{N} G_{M N} \\
& -2 \nabla_{n} x^{M} G_{M u} \nabla_{n} u+\frac{2}{\omega} \partial_{\sigma} x^{M} G_{M u} \partial_{\sigma} u-\nabla_{n} u G_{u u} \nabla_{n} u+\frac{1}{\omega} \partial_{\sigma} u G_{u u} \partial_{\sigma} u \\
& \left.+\lambda^{+}\left(\nabla_{n} u-\frac{1}{\sqrt{\omega}} \partial_{\sigma} u\right) \mathbf{Y}^{+}+\lambda^{-}\left(\nabla_{n} u+\frac{1}{\sqrt{\omega}} \partial_{\sigma} u\right) \mathbf{Y}^{-}+\lambda^{+} \lambda^{-}\right] \\
& -T B_{M N} \partial_{\tau} x^{M} \partial_{\sigma} x^{N}-T \partial_{\tau} x^{M} B_{M u} \partial_{\sigma} u-\partial_{\tau} u B_{u N} \partial_{\sigma} x^{N}
\end{aligned}
$$

where $\mathbf{Y}^{+}=\sqrt{G_{u u}}, \mathbf{Y}^{-}=-\sqrt{G_{u u}}$. In (3.1) wee used $1+1$ form of the world-sheet metric where

$$
\gamma_{\alpha \beta}=\left(\begin{array}{cc}
-N^{2}+N^{\sigma} \omega N^{\sigma} & N^{\sigma} \omega \\
N^{\sigma} \omega & \omega
\end{array}\right), \quad \nabla_{n}=\frac{1}{N}\left(\partial_{\tau}-N^{\sigma} \partial_{\sigma}\right) .
$$

We see from the Lagrangian density that there is exceptional direction labeled with $u$ since solving equations of motion for $\lambda^{+}, \lambda^{-}$and inserting back to the Lagrangian density (3.1) we obtain following contribution

$$
-\left[\nabla_{n} u \nabla_{n} u-\frac{1}{\omega} \partial_{\sigma} u \partial_{\sigma} u\right] \mathbf{Y}^{+} \mathbf{Y}^{-}=\left[\nabla_{n} u \nabla_{n} u-\frac{1}{\omega} \partial_{\sigma} u \partial_{\sigma} u\right] G_{u u}
$$

so that terms proportional to $\left(\nabla_{n} u\right)^{2}$ and $\left(\partial_{\sigma} u\right)^{2}$ are zero and hence the Lagrangian density effectively describes motion of the string in the background with light-like isometry. On the hand we can consider Lagrangian (3.1) in its own and discuss how its properties under T-duality depend on values of $\mathbf{y}^{+}, \mathbf{Y}^{-}$. However the advantage of the Lagrangian (3.1) is that it contains term quadratic in time derivative of $u$ and hence we can easily perform canonical analysis. This has been done in $[4,11]$ with the result

$$
\begin{aligned}
& H=\frac{N}{2 \sqrt{\omega} T}\left[\pi_{M} G^{M N} \pi_{N}+2 \pi_{M} G^{M u}\left(\pi_{u}+\frac{T}{2} \tilde{\lambda}^{+} \mathbf{Y}^{+}+\frac{T}{2} \tilde{\lambda}^{-} \mathbf{Y}^{-}\right)\right. \\
& +\left(\pi_{u}+\frac{T}{2} \tilde{\lambda}^{+} \mathbf{Y}^{+}+\frac{T}{2} \tilde{\lambda}^{-} \mathbf{Y}^{-}\right) G^{u u}\left(\pi_{u}+\frac{T}{2} \tilde{\lambda}^{+} \mathbf{Y}^{+}+\frac{T}{2} \tilde{\lambda}^{-} \mathbf{Y}^{-}\right) \\
& +T^{2} \partial_{\sigma} x^{M} G_{M N} \partial_{\sigma} x^{N}+2 T^{2} \partial_{\sigma} x^{M} G_{M u} \partial_{\sigma} u+T^{2} \partial_{\sigma} u G_{u u} \partial_{\sigma} u \\
& \left.-T^{2} \tilde{\lambda}^{+} \partial_{\sigma} u \mathbf{Y}^{+}+T^{2} \tilde{\lambda}^{-} \partial_{\sigma} u \mathbf{Y}^{-}+T^{2} \tilde{\lambda}^{+} \tilde{\lambda}^{-}\right]+N^{\sigma} \mathcal{H}_{\sigma} \\
& =N^{\tau} \mathcal{H}_{\tau}+N^{\sigma} \mathcal{H}_{\sigma} .
\end{aligned}
$$


where we performed rescaling

$$
\sqrt{\omega} \lambda^{+}=\tilde{\lambda}^{+}, \quad \sqrt{\omega} \lambda^{-}=\tilde{\lambda}^{-}
$$

and in the final step also $N^{\tau}=\frac{N}{2 \sqrt{\omega} T}$. Further,

$$
\pi_{M}=p_{M}+T B_{M N} \partial_{\sigma} x^{N}+T B_{M u} \partial_{\sigma} u, \quad \pi_{u}=p_{u}+T B_{u M} \partial_{\sigma} x^{M} .
$$

It is convenient to introduce common notation $\hat{M}=(M, u)$ so that the Hamiltonian constraint has the form

$$
\begin{aligned}
\mathcal{H}_{\tau}= & \left(\pi_{\hat{M}}+\frac{T}{2} \tilde{\lambda}^{+} \mathbf{Y}_{\hat{M}}^{+}+\frac{T}{2} \tilde{\lambda}^{-} \mathbf{Y}_{\hat{M}}^{-}\right) G^{\hat{M} \hat{N}}\left(\pi_{\hat{N}}+\frac{T}{2} \tilde{\lambda}^{+} \mathbf{Y}_{\hat{N}}^{+}+\frac{T}{2} \tilde{\lambda}^{-} \mathbf{Y}_{\hat{N}}^{-}\right) \\
& +T^{2} \partial_{\sigma} \tilde{x}^{\hat{M}} G_{\hat{M} \hat{N}} \partial_{\sigma} x^{\hat{N}}-T^{2} \tilde{\lambda}^{+} \partial_{\sigma} \tilde{x}^{\hat{M}} \mathbf{Y}_{\hat{M}}^{+}+T^{2} \tilde{\lambda}^{-} \partial_{\sigma} \tilde{x}^{\hat{M}} \mathbf{Y}_{\hat{M}}^{-}+T^{2} \tilde{\lambda}^{+} \tilde{\lambda}^{-},
\end{aligned}
$$

where we also introduced $\mathbf{Y}_{\hat{M}}^{ \pm}=(\overbrace{0, \ldots, 0}^{d}, \mathbf{Y}^{ \pm})$.

We showed in $[4,11]$ that performing T-duality along $u$-direction we obtain nonrelativistic string in torsional Newton-Cartan geometry. We further analyzed T-duality of non-relativistic string along spatial direction in [4] where we were not able to study it in the most general case of non-zero NSNS two form field. Now we fill this gap and consider T-duality transformations along $k$-spatial directions that we label with $y^{i}$. Then T-dual coordinates are related to the original ones by following relations

$$
p_{i}=-T \partial_{\sigma} \tilde{y}_{i}, \quad \tilde{p}^{i}=-T \partial_{\sigma} y^{i}, \quad p_{u}=-T \partial_{\sigma} \eta, \quad \tilde{p}_{\eta}=-T \partial_{\sigma} u .
$$

In what follows we introduce common notation where $\tilde{x}_{m}=\left(\tilde{y}_{i}, \eta\right), \tilde{p}^{m}=\left(\tilde{p}^{i}, \tilde{p}_{\eta}\right)$ and $\mathbf{Y}_{m}^{ \pm}=(\overbrace{0, \ldots, 0}^{k}, \mathbf{Y}^{ \pm})$. Further, remaining coordinates are denoted as $x^{\mu}$ together with conjugate momenta $p_{\mu}$. Then after T-duality transformation we get

$$
\begin{aligned}
& \pi_{\mu}=k_{\mu}-B_{\mu m} \tilde{p}^{m}, \\
& \pi_{i}=-T \partial_{\sigma} \tilde{y}_{i}-B_{i m} \tilde{p}^{m}+T B_{i \mu} \partial_{\sigma} x^{\mu}+\frac{T}{2} \tilde{\lambda}^{+} \mathbf{Y}_{i}^{+}+\frac{T}{2} \tilde{\lambda}^{-} \mathbf{Y}_{i}^{-}=-B_{i m} \tilde{p}^{m}+T \mathbf{V}_{i}, \\
& \pi_{u}=-T \partial_{\sigma} \eta-B_{u m} \tilde{p}^{m}+T B_{u \mu} \partial_{\sigma} x^{\mu}+\frac{T}{2} \tilde{\lambda}^{+} \mathbf{Y}_{i}^{+}+\frac{T}{2} \tilde{\lambda}^{-} \mathbf{Y}_{i}^{-}=-B_{u m} \tilde{p}^{m}+T \mathbf{V}_{u}
\end{aligned}
$$

or equivalently

$$
\pi_{n}=T \mathbf{V}_{n}-B_{n m} \tilde{p}^{m}, \mathbf{V}_{n}=-\partial_{\sigma} \tilde{x}_{n}+B_{n \mu} \partial_{\sigma} x^{\mu}+\frac{1}{2} \tilde{\lambda}^{+} \mathbf{Y}_{n}^{+}+\frac{1}{2} \tilde{\lambda}^{-} \mathbf{Y}_{n}^{-} .
$$

Then T-dual Hamiltonian constraint has the form

$$
\begin{aligned}
\mathcal{H}_{\tau}^{T}= & k_{\mu} G^{\mu \nu} k_{\nu}+\tilde{p}^{m} \mathbf{H}_{m n} \tilde{p}^{n}+2 \tilde{p}^{m} B_{m \hat{M}} G^{\hat{M} \mu} k_{\mu}+2 T k_{\mu} G^{\mu n} \mathbf{V}_{n}+T^{2} \mathbf{V}_{m} G^{m n} \mathbf{V}_{n} \\
& +2 T \tilde{p}^{m}\left(B_{m \hat{M}} G^{\hat{M} n} \mathbf{V}_{n}-G_{m \mu} \partial_{\sigma} x^{\mu}+\frac{1}{2} \tilde{\lambda}^{+} \mathbf{Y}_{m}^{+}-\frac{1}{2} \tilde{\lambda}^{-} \mathbf{Y}_{m}^{-}\right) \\
& +T^{2} \partial_{\sigma} x^{\mu} G_{\mu \nu} \partial_{\sigma} x^{\nu}+T^{2} \tilde{\lambda}^{+} \tilde{\lambda}^{-}
\end{aligned}
$$


where

$$
\mathbf{H}_{m n}=G_{m n}-B_{m \hat{M}} G^{\hat{M} \hat{N}} B_{\hat{N} n} .
$$

Let us now proceed to the Lagrangian formulation of given theory. Since the analysis is completely the same as in case of the relativistic theory we immediately write the result

$$
\begin{aligned}
\mathcal{L}= & \tilde{p}^{m} \partial_{\tau} \tilde{x}_{m} \tilde{p}^{m}+p_{\mu} \partial_{\tau} x^{\mu}-H \\
= & \frac{1}{4 N^{\tau}}\left[g_{\tau \tau}^{\prime}-2 N^{\sigma} g_{\tau \sigma}^{\prime}+\left(N^{\sigma}\right)^{2} g_{\sigma \sigma}^{\prime}\right]-N^{\tau} T^{2} g_{\sigma \sigma}^{\prime}-T b_{\tau \sigma}^{\prime} \\
& -\frac{T}{2} N^{\tau} \tilde{\lambda}^{+}\left[\nabla_{n} \tilde{x}_{m} \mathbf{A}^{m}-2 T \partial_{\sigma} \tilde{x}_{m} \mathbf{A}^{m}+\nabla_{n} x^{\mu} \mathbf{A}_{\mu}-2 T \partial_{\sigma} x^{\mu} \mathbf{A}_{\mu}\right] \\
& -\frac{T}{2} N^{\tau} \tilde{\lambda}^{-}\left[\nabla_{n} \tilde{x}_{m} \mathbf{B}^{m}+2 T \partial_{\sigma} \tilde{x}_{m} \mathbf{B}^{m}+\nabla_{n} x^{\mu} \mathbf{B}_{\mu}+2 T \partial_{\sigma} x^{\mu} \mathbf{B}_{\mu}\right] \\
& -N^{\tau} T^{2} \tilde{\lambda}^{+} \tilde{\lambda}^{-} \mathbf{X},
\end{aligned}
$$

where

$$
\begin{aligned}
\mathbf{A}^{m} & =\tilde{G}^{m n}\left(\mathbf{Y}_{n}^{+}+B_{n k} \hat{G}^{k l} \mathbf{Y}_{l}^{+}\right), & & \mathbf{A}_{\mu}=\left(B_{\mu m}-G_{\mu m}\right) \tilde{G}^{m n}\left(\mathbf{Y}_{n}^{+}+B_{n k} \hat{G}^{k l} \mathbf{Y}_{l}^{+}\right), \\
\mathbf{B}^{m} & =\tilde{G}^{m n}\left(-\mathbf{Y}_{n}^{-}+B_{n l} \hat{G}^{l k} \mathbf{Y}_{k}^{-}\right), & & \mathbf{B}_{\mu}=\left(G_{\mu m}+B_{\mu m}\right) \tilde{G}^{m n}\left(-\mathbf{Y}_{n}^{-}+B_{k l} \hat{G}^{l k} \mathbf{Y}_{n}^{-}\right), \\
\mathbf{X} & =\mathbf{Y}_{m}^{+} \tilde{G}^{m n} \mathbf{Y}_{n}^{-}+1 . & &
\end{aligned}
$$

The nature of the resulting T-dual string depends on the fact whether $\mathbf{X}$ vanishes or not. In case when $\mathbf{X} \neq 0$ we can solve the equation of motion for $\tilde{\lambda}^{+}, \tilde{\lambda}^{-}$as

$$
\begin{aligned}
& -\frac{1}{2}\left[\nabla_{n} \tilde{x}_{m} \mathbf{A}^{m}-2 T \partial_{\sigma} \tilde{x}_{m} \mathbf{A}^{m}+\nabla_{n} x^{\mu} \mathbf{A}_{\mu}-2 T \partial_{\sigma} x^{\mu} \mathbf{A}_{\mu}\right]-T \tilde{\lambda}^{-} \mathbf{X}=0, \\
& -\frac{1}{2}\left[\nabla_{n} \tilde{x}_{m} \mathbf{B}^{m}+2 T \partial_{\sigma} \tilde{x}_{m} \mathbf{B}^{m}+\nabla_{n} x^{\mu} \mathbf{B}_{\mu}+2 T \partial_{\sigma} x^{\mu} \mathbf{B}_{\mu}\right]-T \tilde{\lambda}^{+} \mathbf{X}=0 .
\end{aligned}
$$

These equations can be easily solved for $\tilde{\lambda}^{+}$and $\tilde{\lambda}^{-}$. Then inserting these results into (3.13) we obtain

$$
\mathcal{L}^{\prime \prime}=\frac{1}{4 N^{\tau}}\left[g_{\tau \tau}^{\prime \prime}-2 N^{\sigma} g_{\tau \sigma}^{\prime \prime}+\left(N^{\sigma}\right)^{2} g_{\sigma \sigma}^{\prime \prime}\right]-N^{\tau} T^{2} g_{\sigma \sigma}^{\prime \prime}-T b_{\tau \sigma}^{\prime \prime}
$$

where

$$
\begin{aligned}
& g_{\alpha \beta}^{\prime \prime}=G^{\prime \prime m n} \partial_{\alpha} \tilde{x}_{m} \partial_{\beta} \tilde{x}_{n}+G_{\nu}^{\prime \prime m} \partial_{\alpha} \tilde{x}_{m} \partial_{\beta} x^{\nu}+G_{\mu}^{\prime \prime n} \partial_{\alpha} x^{\mu} \partial_{\beta} \tilde{x}_{n}+G_{\mu \nu}^{\prime \prime} \partial_{\alpha} x^{\mu} \partial_{\beta} x^{\nu}, \\
& b_{\tau \sigma}^{\prime \prime}=B^{\prime \prime m n} \partial_{\tau} \tilde{x}_{m} \partial_{\sigma} \tilde{x}_{n}+B_{\nu}^{\prime \prime m} \partial_{\tau} \tilde{x}_{m} \partial_{\sigma} x^{\nu}+B_{\mu}^{\prime \prime} \partial_{\tau} x^{\mu} \partial_{\sigma} \tilde{x}_{n}+B_{\mu \nu}^{\prime \prime} \partial_{\tau} x^{\mu} \partial_{\sigma} x^{\nu},
\end{aligned}
$$

where primed components of the background metric and NSNS two form have the form

$$
\begin{aligned}
G^{\prime \prime m n} & =G^{\prime m n}+\frac{1}{2 \mathbf{X}}\left(\mathbf{A}^{m} \mathbf{B}^{m}+\mathbf{A}^{n} \mathbf{B}^{m}\right), & G_{\mu}^{\prime \prime m} & =G_{\mu}^{\prime m}+\frac{1}{2 \mathbf{X}}\left(\mathbf{A}^{m} \mathbf{B}_{\mu}+\mathbf{B}^{m} \mathbf{B}_{\mu}\right), \\
G_{\mu \nu}^{\prime \prime} & =G_{\mu \nu}^{\prime}+\frac{1}{2 \mathbf{X}}\left(\mathbf{A}_{\mu} \mathbf{B}_{\nu}+\mathbf{A}_{\nu} \mathbf{B}_{\mu}\right), & B^{\prime \prime m n} & =B^{\prime m n}+\frac{1}{2 \mathbf{X}}\left(\mathbf{B}^{m} \mathbf{A}^{n}-\mathbf{B}^{n} \mathbf{A}^{m}\right), \\
B_{\mu}^{\prime \prime m} & =B_{\mu}^{\prime m}+\frac{1}{2 \mathbf{X}}\left(\mathbf{B}^{m} \mathbf{A}_{\mu}-\mathbf{A}^{m} \mathbf{B}_{\mu}\right), & B_{\mu \nu}^{\prime \prime} & =B_{\mu \nu}^{\prime}+\frac{1}{2 \mathbf{X}}\left(\mathbf{B}_{\mu} \mathbf{A}_{\nu}-\mathbf{B}_{\nu} \mathbf{A}_{\mu}\right) .
\end{aligned}
$$

Clearly resulting string is relativistic string in the background fields given in (3.18). 
As the second case let us consider the case when $\mathbf{X}=0$ that, according to discussion below (3.1) corresponds to the original string with light-like isometry. In this case the equation of motion for $\tilde{\lambda}^{ \pm}$have the form

$$
\begin{aligned}
& \nabla_{n} \tilde{x}_{m} \mathbf{A}^{m}-2 T \partial_{\sigma} \tilde{x}_{m} \mathbf{A}^{m}+\nabla_{n} x^{\mu} \mathbf{A}_{\mu}-2 T \partial_{\sigma} x^{\mu} \mathbf{A}_{\mu}=0 \\
& \nabla_{n} \tilde{x}_{m} \mathbf{B}^{m}+2 T \partial_{\sigma} \tilde{x}_{m} \mathbf{B}^{m}+\nabla_{n} x^{\mu} \mathbf{B}_{\mu}+2 T \partial_{\sigma} x^{\mu} \mathbf{B}_{\mu}=0 .
\end{aligned}
$$

We multiply first equation with $\partial_{\sigma} \tilde{x}_{m} \mathbf{B}^{m}+\partial_{\sigma} x^{\mu} \mathbf{B}_{\mu}$ and the second one with $\partial_{\sigma} \tilde{x}_{m} \mathbf{B}^{m}+$ $\partial_{\sigma} x^{\mu} \mathbf{B}_{\mu}$ and sum these two equations so that we get

$$
\begin{aligned}
N^{\sigma}= & \frac{\mathbf{a}_{\tau \sigma}}{\mathbf{a}_{\sigma \sigma}}, \\
\mathbf{a}_{\alpha \beta}= & \frac{1}{2} \partial_{\alpha} \tilde{x}_{m}\left(\mathbf{A}^{m} \mathbf{B}^{n}+\mathbf{A}^{n} \mathbf{B}^{m}\right) \partial_{\beta} \tilde{x}_{n}+\frac{1}{2} \partial_{\alpha} \tilde{x}_{m}\left(\mathbf{A}^{m} \mathbf{B}_{\mu}+\mathbf{B}^{m} \mathbf{A}_{\mu}\right) \partial_{\beta} x^{\nu} \\
& +\frac{1}{2} \partial_{\alpha} x^{\mu}\left(\mathbf{A}_{\mu} \mathbf{B}^{m}+\mathbf{B}_{\mu} \mathbf{A}^{m}\right) \partial_{\beta} \tilde{x}_{m}+\frac{1}{2} \partial_{\alpha} x^{\mu}\left(\mathbf{A}_{\mu} \mathbf{B}_{\nu}+\mathbf{A}_{\nu} \mathbf{B}_{\mu}\right) \partial_{\beta} x^{\nu} .
\end{aligned}
$$

On the other hand if we multiply two equations in (3.19) together and use (3.20) we obtain $N^{\tau}$ to be equal to

$$
N^{\tau}=\frac{\sqrt{-\operatorname{det} \mathbf{a}_{\alpha \beta}}}{2 T \mathbf{a}_{\sigma \sigma}} .
$$

Finally we obtain T-dual string in the form of non-relativistic action

$$
S=\int d \tau d \sigma \mathcal{L}
$$

where

$$
\mathcal{L}=-\frac{T}{2} \sqrt{-\operatorname{det} \mathbf{a}} \mathbf{a}^{\alpha \beta} g_{\alpha \beta}^{\prime}-T B_{\tau \sigma}^{\prime}
$$

where $\mathbf{a}^{\alpha \beta}$ is matrix inverse to $\mathbf{a}_{\alpha \beta}$.

Before we proceed to an explicit example let us briefly comment about duality group of non-relativistic string theory. From previous discussion we know that direction $u$ is exceptional since it is light-like. Then we can claim that T-duality group of non-relativistic string when we perform T-duality along $k$-spatial transverse directions is the same as in case of relativistic string which is $O(k, k)$ [1].

Let us be more explicit and consider following background with light-like isometry [5-8]

$$
d s^{2}=g_{\hat{M} \hat{N}} d x^{\hat{M}} d x^{\hat{N}}=2 \tau(d u-m)+h_{M N} d x^{M} d x^{N}, \tau=\tau_{M} d x^{M}, \quad m=m_{M} d x^{M},
$$

where NSNS two form has following components

$$
B_{M N}, B_{u M}=b_{M} .
$$

Let us now consider T-duality along single spatial coordinate that we denote as $y$ together with T-duality along direction labeled by $u$. Then we have

$$
\begin{aligned}
& G_{\mu \nu}=h_{\mu \nu}-\tau_{\mu} m_{\nu}-m_{\nu} \tau_{\mu} \equiv \hat{h}_{\mu \nu}, \\
& G_{u u}, \quad G_{u y}=\tau_{y}, \quad G_{u \mu}=\tau_{\mu}, \\
& G_{y y}=h_{y y}-2 \tau_{y} m_{y}, \quad G_{y \mu}=h_{y \mu}-\tau_{y} m_{\mu}-\tau_{\mu} m_{y}
\end{aligned}
$$


so that the metric $G_{m n}$ and two form $B_{m n}$ have following form

$$
G_{m n}=\left(\begin{array}{cc}
G_{u u} & \tau_{y} \\
\tau_{y} & h_{y y}-2 \tau_{y} m_{y}
\end{array}\right), \quad B_{m n}=\left(\begin{array}{cc}
0 & b_{y} \\
-b_{y} & 0
\end{array}\right)
$$

so that metric inverse to $G_{m n}$ is equal to

$$
\hat{G}^{m n}=\frac{1}{G_{u u}\left(h_{y y}-2 \tau_{y} m_{y}\right)-\tau_{y}^{2}}\left(\begin{array}{cc}
h_{y y}-2 \tau_{y} m_{y}-\tau_{y} \\
-\tau_{y} & G_{u u}
\end{array}\right) .
$$

We would like to consider situation when T-dual string is again non-relativistic string that is ensured when $\mathbf{X}=0$. Since $\mathbf{Y}_{u}^{ \pm}= \pm \sqrt{G_{u u}}$ we see that in order to ensure this condition we should have $\tilde{G}^{u u}=\frac{1}{G^{u u}}$. In order to ensure this requirement we firstly impose condition that $\tau_{y}=0$ so that the matrices $\hat{G}_{m n}$ and $\tilde{G}^{m n}$ have the form

$$
\hat{G}_{m n}=\left(\begin{array}{cc}
G_{u u}-\frac{b_{y} b_{y}}{G_{y y}} & 0 \\
0 & G_{y y}-\frac{b_{y} b_{y}}{G_{u u}}
\end{array}\right), \quad \tilde{G}^{m n}=\left(\begin{array}{cc}
\frac{1}{G_{u u}-\frac{b_{y} b_{y}}{G_{y y}}} & 0 \\
0 & \frac{1}{G_{y y}-\frac{b_{y} b_{y}}{G_{u u}}}
\end{array}\right)
$$

and hence $\mathbf{X}=0$ on condition that $b_{y}=0$. Then

$$
\begin{array}{rlrl}
\mathbf{A}^{u} & =\frac{1}{\sqrt{G_{u u}}}, & \mathbf{A}^{y}=0, & \mathbf{A}_{\mu}=-\left(b_{\mu}+\tau_{\mu}\right) \frac{1}{\sqrt{G_{u u}}}, \\
\mathbf{B}^{u}=\frac{1}{\sqrt{G_{u u}}}, & \mathbf{B}^{y}=0, & \mathbf{B}_{u}=\left(\tau_{\mu}-b_{\mu}\right) \frac{1}{\sqrt{G_{u u}}}
\end{array}
$$

and hence we get

$$
\mathbf{a}_{\alpha \beta}=\frac{1}{G_{u u}}\left(\left(\partial_{\alpha} \eta-b_{\alpha}\right)\left(\partial_{\beta} \eta-b_{\beta}\right)-\tau_{\alpha} \tau_{\beta}\right) .
$$

Finally we obtain transformation rules for components of the metric

$$
\begin{array}{rlrl}
G_{\mu \nu}^{\prime} & =\hat{h}_{\mu \nu}-\frac{1}{G_{u u}} \tau_{\mu} \tau_{\nu}-\frac{\hat{h}_{\mu y} \hat{h}_{y \nu}}{G_{y y}}+\frac{1}{G_{u u}} b_{\mu} b_{\nu}-\frac{1}{G_{y y}} B_{\mu y} B_{y \nu} \\
& =\hat{h}_{\mu \nu}^{\prime}+\frac{1}{G_{u u}}\left(b_{\mu} b_{\nu}-\tau_{\mu} \tau_{\nu}\right), & \\
G_{\eta \eta}^{\prime} & =\frac{1}{G_{u u}}, & G_{\tilde{y} \tilde{y}}^{\prime}=\frac{1}{G_{y y}}, \\
G_{\eta \nu}^{\prime} & =-\frac{b_{\nu}}{G_{u u}}=G_{\nu \eta}^{\prime}, & G_{\tilde{y} \mu}=-\frac{1}{G_{y y}} B_{y \mu}=G_{\mu \tilde{y}}, \\
B_{\mu \nu}^{\prime} & =B_{\mu \nu}+\frac{1}{G_{u u}}\left(\tau_{\mu} b_{\nu}-b_{\mu} \tau_{\nu}\right)+\frac{1}{G_{y y}}\left(\hat{h}_{\mu y} B_{y \nu}-B_{\mu y} \hat{h}_{y \nu}\right), \\
B_{\mu \tilde{y}}^{\prime} & =\frac{G_{\mu y}}{G_{y y}}, & B_{\mu \eta}^{\prime}=\frac{\tau_{\mu}}{G_{u u}} .
\end{array}
$$

These are generalization of the T-duality rules that were determined in our previous paper [4] to the case of non-zero NSNS two form. We also see that T-duality of non-relativistic string is again non-relativistic string when we impose restriction on the background metric given by conditions $\tau_{y}=b_{y}=0$ which agrees with the condition found in [4]. 
Open Access. This article is distributed under the terms of the Creative Commons Attribution License (CC-BY 4.0), which permits any use, distribution and reproduction in any medium, provided the original author(s) and source are credited.

\section{References}

[1] A. Giveon, M. Porrati and E. Rabinovici, Target space duality in string theory, Phys. Rept. 244 (1994) 77 [hep-th/9401139] [INSPIRE].

[2] T.H. Buscher, A Symmetry of the String Background Field Equations, Phys. Lett. B 194 (1987) 59 [INSPIRE].

[3] T.H. Buscher, Path Integral Derivation of Quantum Duality in Nonlinear $\sigma$-models, Phys. Lett. B 201 (1988) 466 [INSPIRE].

[4] J. Kluso n, T-duality of Non-Relativistic String in Torsional Newton-Cartan Background, JHEP 05 (2020) 024 [arXiv: 1909.13508] [INSPIRE].

[5] T. Harmark, J. Hartong and N.A. Obers, Nonrelativistic strings and limits of the AdS/CFT correspondence, Phys. Rev. D 96 (2017) 086019 [arXiv: 1705.03535] [INSPIRE].

[6] J. Kluso n, Remark About Non-Relativistic String in Newton-Cartan Background and Null Reduction, JHEP 05 (2018) 041 [arXiv: 1803.07336] [INSPIRE].

[7] T. Harmark, J. Hartong, L. Menculini, N.A. Obers and Z. Yan, Strings with Non-Relativistic Conformal Symmetry and Limits of the AdS/CFT Correspondence, JHEP 11 (2018) 190 [arXiv: 1810.05560] [INSPIRE].

[8] T. Harmark, J. Hartong, L. Menculini, N.A. Obers and G. Oling, Relating non-relativistic string theories, JHEP 11 (2019) 071 [arXiv:1907.01663] [INSPIRE].

[9] E. Alvarez, L. Álvarez-Gaumé and Y. Lozano, A canonical approach to duality transformations, Phys. Lett. B 336 (1994) 183 [hep-th/9406206] [INSPIRE].

[10] E. Alvarez, L. Álvarez-Gaumé and Y. Lozano, An introduction to T duality in string theory, hep-th/9410237 [INSPIRE].

[11] J. Kluso` n, Note About Canonical Description of T-duality Along Light-Like Isometry, Eur. Phys. J. C 80 (2020) 103 [arXiv: 1905.12910] [inSPIRE].

[12] E. Bergshoeff, J. Gomis and Z. Yan, Nonrelativistic String Theory and T-duality, JHEP 11 (2018) 133 [arXiv: 1806.06071] [INSPIRE]. 